\title{
CARACTERISTICAS DE LOS SUELOS Y CAPACIDAD DE USO MAYOR DE LAS TIERRAS DE LA RESERVA NACIONAL PACAYA - SAMIRIA*
}

\author{
Fernando Rodríguez Achung ** \\ Roger Escobedo T orres ** \\ J uan Ramírez Barco ** \\ León Bendayán A costa ** \\ Carlos R ojas Rodríguez ** \\ Carlos C alle Barco** \\ Roger Riva Rodríguez ** \\ L uis Marquina Picón **
}

\section{RESUMEN}

En la Reserva Nacional Pacaya Samiria y en las áreas colindantes, que cubre un área de 3'236,878 ha, se ha realizado un estudio de evaluación de los suelos con fines de conservación y desarrollo.

Cerca del $57 \%$ del área estudiada, está constituida por terrazas bajas con diferentes grados de drenaje, seguido por los complejos de orillares con el $20 \%$. $\mathrm{L}$ as terrazas medias y las colinas y lomadas bajas, en conjunto sólo representan el $18 \%$.

Predominan suelos jóvenes de incipiente desarrollo genético formados a partir de sedimentos recientes y subrecientes de aceptable fertilidad natural y aptitud agrícola estacional, y suelos pobres, especialmente aquellos derivados de materiales residuales y sedimentos aluviales antiguos.

De acuerdo con el Soil Taxonomy se ha logrado identificar ocho grandes grupos de suelos: Hydraquents, Fluvaquents, Tropofluvents, Tropaquepts, Eutropepts, Dystropepts, Hapludults y Tropofibrist, siendo los más abundantes aquellos que pertenecen a los Ordenes Entisol e Inceptisol.

Estudio realizado en el marco del convenio IIAP-FPCN-CDC.

Investigadores del IIAP. 
Según la Clasificación de Capacidad de U so M ayor, el potencial del área estudiada es el siguiente:

- Tierras aptas para cultivo en limpio, perteneciente a la subclase: A3i 280,575 has $(8.7 \%)$.

- Tierras aptas para cultivo permanente, perteneciente a las subclases C3s y C3se 149,690 ha $(4.6 \%)$.

- Tierras aptas para producción forestal, perteneciente a las subclases F 2w, F 2se, F 3w, F 3sw 1' 936,421 ha (59.8\%).

- Tierras de protección, incluyendo ríos, meandros, lagunas y localidades 870,192 ha $(26.9 \%)$

\section{INTRODUCCION}

La Reserva Nacional Pacaya Samiria y su zona de influencia, por sus características físicas y biológicas, desempeña un papel importante en la economía y en la conservación de una parte significativa de la Región A mazónica y es una de las más importantes áreas de conservación del país (Rodríguez, Vásquez y Rodríguez, 1995).

En el área estudiada existen 77 mil personas que hacen uso directo de los recursos naturales de la Reserva y que se caracterizan por su bajo nivel de vida.

La población, a parte de las actividades de caza, pesca y extracción, desarrolla una actividad agrícola que se localiza fundamentalmente en las áreas inundables de los ríos M arañón y U cayali/Canal de Puinahua, y que se caracteriza por ser estacional, de pequeña escala, intensiva en el uso de la mano de obra, de rendimiento bajo y por el empleo de tecnologías tradicionales (IIAP, 1994).

Con el objeto de compatibilizar el desarrollo de las comunidades ribereñas y la conservación de esta Reserva $\mathrm{Nacional}$, en el marco del proyecto "Employment and Natural Resources Sustainability № 527-0341" ejecutado por la Fundación Peruana para la Conservación de la Naturaleza (FPCN), con el apoyo de The Nature Conservancy (TNC) y el US-A gency for the International Development (US-AID), se ha priorizado diversos estudios básicos, dentro del 
cual se circunscribe el presente estudio de evaluación de suelos y de la capacidad de uso de la tierra.

El estudio se ha realizado en dos fases, la primera cubre una superficie de 632,796 has, ubicada en la parte mas septentrional de la Reserva Nacional y que se ejecutó entre los meses de octubre de 1992 y marzo de 1993.

L a segunda fase, que cubre el resto de área de dicha Reserva, se ejecutó entre los meses de julio de 1993 y febrero de 1994.

\section{MATERIALES Y METODOS}

\subsection{C aracterísticas G enerales de la Zona en E studio}

La zona estudiada se encuentra situada en el sector Nororiental del Perú, dentro de la selva baja, cubriendo todo el territorio de la Reserva $\mathrm{N}$ acional Pacaya - Samiria (RNPS), incluyendo áreas adyacentes de amortiguamiento. Políticamente se ubica principalmente en las provincias de Loreto y Requena, pertenecientes a la región L oreto.

L a zona comprende parte de la cuenca inferior de los ríos U cayali y M arañón, los que confluyen en su extremo Nororiental para dar nacimiento al A mazonas.

A simismo, presentan dos pequeñas subcuencas, conformadas por los ríos Pacaya y Samiria; se aprecia también la existencia de tres (3) quebradas, denominadas $\mathrm{Y}$ anayacu del Pucate, Pucate y Nauta caño. El área total estudiada es de 3' 236,878 has.

De acuerdo al Mapa Ecológico del Perú, la zona de estudio se encuentra comprendida en la zona de vida de Bosque Húmedo Tropical (Bh-T).

La temperatura media mensual varía de 20 a $33^{\circ} \mathrm{C}$ y la precipitación anual promedio varía de 2,000 a $4,000 \mathrm{~mm}$.

La Reserva Nacional Pacaya Samiria, desde el punto de vista morfoestructural, está constituida por dos sectores muy nítidos: el primero, que es la más grande, conforma una gran depresión estructural, denominada depresión U camara, derivada de los ríos U cayali y M arañón que la bordean y constituida por depósitos inconsolidados del cuaternario y la segunda, ubicada 
en el borde Suroccidental está conformada principalmente por un frente de colinas del terciario.

Procesos morfodinámicos, de erosión lateral y sedimentación, debido a la dinámica de los ríos, caracterizan a la zona de estudio.

\subsection{Material Cartográfico}

EI material cartográfico utilizado en el presente estudio, fue el siguiente:

- Mapa preliminar de vegetación, elaborado en base al análisis digital de imágenes LANDSAT-TM, por el CDC de la Universidad Agraria La Molina.

- M osaicos aerofotográficos controlados a escala 1:40,000; año 1957.

- Fotografías aéreas pancromáticas en blanco y negro a escala 1:40,000; año 1957.

- F otocartas nacionales del departamento de L oreto a escala 1:100,000.

- M apas de la Reserva N acional del Pacaya Samiria, a escala 1:400,000.

- Mapa fisiográfico de la zona Boca del Pachitea - Nauta, a escala $1: 100,000$.

- Mapas topográficos e hidrográficos en la zona de estudio a escala $1: 100,000$.

\subsection{M etodología}

El estudio se ejecutó en cuatro etapas :

\section{a) Etapa preliminar de G abinete}

En esta etapa se realizó la recopilación de la información necesaria sobre los trabajos existentes dentro del área, también se elaboró el mapa base 
fisiográfico-edáfico que ha sido utilizado en el planeamiento de los trabajos de la etapa de campo.

\section{b) Etapa de Campo}

En la primera fase del estudio, se ha realizado un vuelo de reconocimiento, con el objeto de verificar las unidades fisiográficas del mapa base.

Para la ejecución del trabajo de campo se seleccionaron veintidós zonas de muestreo, donde se realizaron trochas para la verificación y corrección de las unidades fisiográficas, así como para realizar el muestreo y descripción de los suelos, mediante la apertura de calicatas y sondeos por barrenajes.

La descripción de perfiles y colección de muestras de suelos se realizaron en siete zonas de muestreo, de acuerdo al detalle siguiente:

Zona de muestreo

Barrenajes de Calicatas

Sondeos Descrit. Descritas
Node

M uestras

$\begin{array}{lrrrr}\text { Y anayacu-Pucate } & 22 & 1 & 6 & 29 \\ \text { San Pablo de Tipishca } & 7 & 1 & 5 & 18 \\ \text { Bagazán } & 2 & 2 & 6 & 14 \\ \text { Caucho grande- Cauchillo } & 11 & 1 & 4 & 24 \\ \text { Hamburgo } & 13 & 2 & 1 & 8 \\ \text { Ungurahui-Y anayacu grande } & 12 & 1 & --- & --- \\ \text { Base Tacshacocha } & 12 & --- & 1 & 5 \\ \text { San M artín Leoncio Prado } & 6 & --- & 4 & 17 \\ \text { Santa Rosa - Lagunas } & 6 & --- & 4 & 17 \\ \text { Nueva Esperanza } & 9 & 1 & 4 & 10 \\ \text { Roca Fuerte - Ollanta } & 7 & 4 & 1 & 5 \\ \text { J enaro Herrera } & 3 & 6 & 7 & 17\end{array}$


Requena

Caro Curahuayte

$M$ anco Capac

Alfaro

Santa Cruz

Tamara

W acra chiro

Victoria

Juancito

M onte bello
2

4

1

5

1

2

4

3

2

2

$--$

1

2

5

1

2

--.

3

---

3
10

13

42

19

13

9

12

7

24

14

TOTAL

\section{c) Etapa de Laboratorio}

Esta etapa consistió en la preparación y análisis de las muestras de suelos descritos en los perfiles modales, los cuales fueron realizados en el laboratorio de suelos del IIAP.

\section{d) E tapa final de Gabinete}

Esta etapa comprendió el procesamiento final y la compilación de la información de campo y laboratorio, así como el reajuste necesario de la fotointerpretación preliminar y el trazo definitivo de las unidades de mapeo, describiéndolos en base a su morfología y resultados de los análisis de laboratorio. A demás se hizo la interpretación de las unidades edáficas, identificadas, en cuanto a la aptitud potencial, simbología y representación gráfica. 


\section{RESULTADOS Y DISCUSION}

\subsection{Evaluación de la Capacidad de Uso de la Tierra}

Estudios realizados por Hoag (1985), en áreas inundables de los ríos tributarios del A mazonas en el Perú, sugieren que el nivel de fertilidad de los suelos es controlado por las características litológicas de la formación geológica donde se origina el tributario y que la ocurrencia y distribución de los suelos contrastantes es predecible.

En la Reserva Nacional Pacaya Samiria (RN PS) y en las zonas colindantes, se registra la misma tendencia. Los suelos que poseen mayor fertilidad están constituidos por depósitos de material sedimentario transportado por los ríos $M$ arañón, Huallaga y U cayali, que tienen su nacimiento en las montañas andinas, donde predominan depósitos de origen marino emergentes, que contienen calizas y areniscas de característica cal cárea continental.

En cambio, los suelos de menor fertilidad se encuentran en áreas que no soportan la influencia de estos ríos, ubicados mayormente fuera de la Reserva, o que son alimentados por pequeños ríos como el Samiria, Pacaya, Pucate y el $Y$ anayacu, que nacen en la misma Reserva. Estos suelos son extremadamente ácidos y de baja capacidad para fines agrícolas.

Esta situación se refleja al analizar la capacidad de uso mayor de las tierras, es decir su utilización óptima permisible que corresponde a sus características ecológicas intrínsicas, tal como lo establece el Reglamento Peruano sobre la clasificación de tierras y sus ampliaciones realizadas por la ONERN.

Sobre el particular, cabe recordar que el objetivo principal en la clasificación de tierras según su capacidad de uso mayor, es determinar el uso adecuado para fines agrícolas, pecuarios, forestales o de protección; así como definir las prácticas de manejo y conservación a fin de evitar su deterioro.

En términos generales, se puede apreciar que de toda el área estudiada (3'236,878 has), las tierras aptas para producción forestal y protección, dominan el escenario agrológico, ya que ambas categorías cubren cerca del $87 \%$ de la superficie, y sólo el $13 \%$ son tierras aptas para actividades agrícolas. 
Sin embargo, el nivel de participación de las tierras para cultivo en limpio $(9 \%)$ y de las tierras para cultivo permanente $(4 \%)$ en la zona de estudio, es muy superior al reportado, tanto para el departamento de L oreto como para el total nacional (ONERN, 1982) (Cuadro No 1).

\section{C uadro № 1 Distribución de las tierras según capacidad de uso mayor a nivel nacional, regional y en la zona de estudio}

\begin{tabular}{|c|c|c|c|c|c|c|}
\hline \multirow{2}{*}{$\begin{array}{l}\text { CAPACIDAD DE US } \\
\text { MAYOR }\end{array}$} & \multicolumn{2}{|c|}{ PERU } & \multicolumn{2}{|c|}{ LORETO } & \multicolumn{2}{|c|}{ RNPS (*) } \\
\hline & ha & $\%$ & ha & $\%$ & ha & $\%$ \\
\hline Cultivo en limpio & $4^{\prime} 902,000$ & 4.0 & 540,000 & 2.0 & 280,575 & 9.0 \\
\hline Cultivo permanente & $2^{\prime} 707,000$ & 2.0 & 607,000 & 2.0 & 149,690 & 4.0 \\
\hline Pastos & $17^{\prime} 916,000$ & 14.0 & $2^{\prime} 229,000$ & 6.0 & $-\cdots \cdot-\cdot-\cdot$ & $-\cdot-\cdot$ \\
\hline Producción Forestal & $48^{\prime} 696,000$ & 38.0 & $27^{\prime} 615,000$ & 80.0 & $1^{\prime} 936,421$ & 60.0 \\
\hline Protección & $54^{\prime} 300,560$ & 42.0 & $3^{\prime} 465,100$ & 10.0 & 870,192 & 27.0 \\
\hline TOTAL & $128^{\prime} 521,560$ & 100.0 & $34^{\prime} 456,100$ & 100.0 & $3^{\prime} 236,876$ & 100.0 \\
\hline
\end{tabular}

$\left(^{*}\right)$ Zona en estudio

En cuanto al patrón de distribución espacial de las tierras para cultivo en limpio, se han hallado las mismas tendencias reportadas por Hoag (1985), concentrándose las mejores tierras, en la planicie inundable por los ríos U cayali, Marañón y Huallaga, y una pequeña parte en el interior de la Reserva, correspondientes a antiguos cauces del U cayali, que hoy en día son recorridas por otros ríos.

De igual modo, del total de tierras aptas para cultivo en limpio, cerca del $70 \%$ se localizan dentro de la jurisdicción de la RNPS, mientras que del total de tierras aptas para cultivo permanente sólo el $55 \%$ se ubican en la RNPS, concentrándose fundamentalmente en la margen derecha del río U cayali, entre Requena y Bagazán, en la margen izquierda del río M arañón, entre San Regis 
y Nauta, y en el límite occidental de la Reserva que abarca la zona comprendida entre Veracruz en el río Marañón y Santa Rosita en el río U cayali ( Cuadro $\mathrm{N}$ ㅇ) ).

Cuadro № 2 Nivel de participación de las zonas internas y externas de la R.N.P.S. En la superficie de las tierras según su capacidad de uso mayor

CAPACIDAD DE

USO MAYOR
TOTAL

AREA

ESTUDIO
NIVEL DE PARTICIPACION

ZONA INTERNA ZONA EXTERNA

ha $\%$ ha $\%$
Cultivo en limpio

Cultivo permanente

Producción Forestal

Protección
280,575

149,690

$1^{\prime} 936,421$

870,192
$195,840 \quad 70.0$

$82,121 \quad 55.0$

$1^{\prime} 373,707 \quad 71.0$

$490,218 \quad 56.0$

66.0

$84,735 \quad 30.0$

$67,569 \quad 45.0$

$562,714 \quad 29.0$

$379,974 \quad 44.0$

L a clasificación a nivel de grupo, clase y subclase de capacidad de uso mayor, para la zona estudiada se detalla a continuación. La superficie y el porcentaje de las tierras identificadas se presentan en el Cuadro $\mathrm{N}$ ㅇ 3 . 


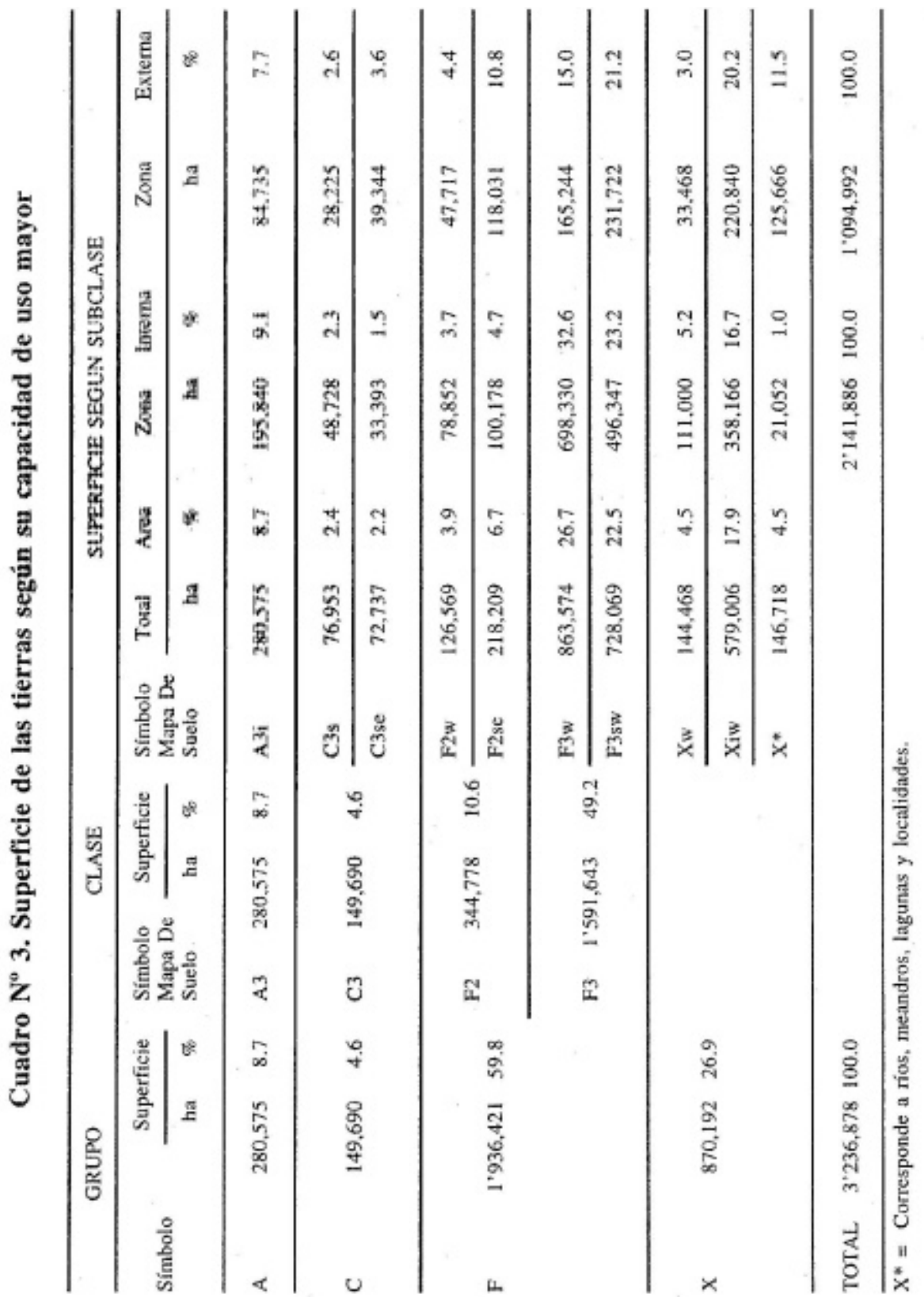




\section{a) Tierras aptas para cultivo en limpio (A)}

Estas tierras comprenden una superficie aproximada de 280,575 ha, que representa el $8.7 \%$ del área total evaluada, e incluye aquellas tierras que presentan las mejores condiciones físicas, químicas y topográficas, donde se puede implantar ampliamente cultivos de corto período vegetativo acorde con las condiciones ecológicas de la zona.

D entro de este grupo, se ha establecido una clase de capacidad de uso (A 3).

\section{CLASE A3}

A grupa a tierras de calidad agrológica baja y apropiada para la explotación agrícola con prácticas intensas de manejo. Estas tierras están sujetas a inundaciones periódicas, y en base a esta limitación se ha determinado la subclase: A $3 i$.

\section{Subclase A3i}

Ocupa una superficie de 280,575 has, que representa el $8.7 \%$ del área total evaluada, de las cuales aproximadamente el $70 \%$ pertenece a la reserva y el $30 \%$ al área de influencia. Incluye a suelos moderadamente profundos a profundos; con drenaje natural bueno a moderado, con textura media a moderadamente fina, de reacción moderadamente neutra a ácida, con niveles medios a bajos de fertilidad.

LoS suelos que integran esta categoría son el suelo Gramalote, Lupuna y San Ramón en su fase de unidades fisiográficas: Isla, complejo de orillares reciente, subreciente, antiguo y terraza baja.

Las limitaciones están relacionadas con la inundación que soportan estas áreas en las épocas de creciente de los ríos U cayali y Marañón, principalmente.

De acuerdo a las condiciones edáficas y climáticas se recomienda el cultivo de especies de corto período vegetativo, como son: arroz, maíz, caupí, soya, maní, yuca, plátano, hortalizas como: caihua, ají dulce, culantro, pepino, zapallo, etc. Entre frutales tenemos: papaya, sandía, melón, etc. 
El uso y manejo de estas tierras deben estar sujetas a un plan adecuado de siembra durante el período de vaciante, a fin de que la inundación no afecte el desarrollo de los cultivos y su respectiva cosecha.

\section{b) Tierras aptas para cultivo permanente (C)}

Estas tierras comprenden una superficie de 149,690 ha, que representa el $4.6 \%$ del área total evaluada.

Por sus limitaciones edáficas no permiten la implantación de cultivos en limpio, pero si una agricultura en base a especies permanentes. Dentro de este grupo se ha determinado la clase $\mathrm{C} 3$.

\section{CLASE C 3}

A grupa tierras de calidad agrológica baja adecuadas para la explotación de cultivos permanentes con prácticas intensivas de manejo. Se encuentran ubicadas en terrazas medias esporádicamente inundables y en colinas bajas y lomadas ligeramente disectadas, de baja fertilidad natural. En esta clase se identifican dos subclases: C 3s y C 3se.

\section{Subclase C 3s}

Cubre una superficie de 76,953 ha, que representa el 2.4\% del área total evaluada, encontrándose cerca del $63 \%$ dentro de la reserva, agrupa a tierras de calidad agrológica baja, incluye suelos profundos a moderadamente profundos con drenaje natural pobre a moderado, de textura fina a moderadamente fina, con reacción extremadamente ácida a muy fuertemente ácida.

El suelo Bagazán integra esta categoría en su fase de unidad fisiográfica, denominada terraza media.

L a limitación más importante está referida a su fertilidad natural baja, bajos niveles de fósforo y alta saturación de aluminio y algunas veces al pobre drenaje que exhiben, debido a la cantidad de arcilla que presentan.

En el uso y manejo de estas tierras, deberá dársele prioridad a especies nativas y exóticas, adaptables a las condiciones ecológicas del medio, 
recomendándose especies como caimito, zapote, palta, mango, castaña, taperiba, cocona, guanábana, umarí, cítricos y pan del árbol, entre los más importantes; y algunas especies forestales como tornillo, aceite caspi, almendra, etc.

Las prácticas agronómicas deberán estar orientadas a incrementar 0 mantener la materia orgánica, mediante la incorporación de residuos de cosecha 0 abonos verdes, etc. y establecer sistemas de drenaje cuando sea necesario.

\section{Subclase C 3se}

Cubre una superficie de 72,737 ha., que representa el $2.2 \%$ del área total evaluada, de las cuales aproximadamente el $46 \%$ está dentro de la reserva. Agrupa tierras de calidad agrológica baja, con suelos moderadamente profundos a profundos, con drenaje natural de bueno a moderado, de textura fina a gruesa, con reacción extremadamente ácida a muy fuertemente ácida.

Integra esta categoría el suelo lomada en su fase de unidad fisiográfica de colina ligeramente a moderadamente disectadas y lomadas, con pendientes que varían hasta 15\%, la cual le confiere el riesgo de erosión.

L as prácticas agrícolas, se orientarán a la implantación de cultivos perennes con cobertura de pastos y forrajes bajo el sistema de curvas de nivel, con manejo e incorporación de materia orgánica.

\section{c) Tierras aptas para producción forestal (F)}

Estas tierras comprenden una superficie de 1'936,421 ha, que representa el 59.8\% del área estudiada.

Están conformadas por tierras que tienen limitaciones edáficas, topográficas y de drenaje, y que a la vez son inapropiadas para llevar a cabo la actividad agropecuaria, quedando relegadas para la producción forestal. Se ha delimitado dos clases F 2 y $F 3$. 


\section{CLASE F 2}

Cubre una superficie aproximada de 344,778 ha., que representa el $10.6 \%$ del área total evaluada. A grupa tierras de calidad agrológica media, con limitaciones para la producción forestal, vinculadas al aspecto topográfico y al drenaje. Considerando estas limitaciones se han identificado las subclases F 2w, F 2se.

\section{Subclase F 2w}

Ocupa una superficie de 126,569 has, que representa el 3.9\% del área total evaluada, de las cuales el $62 \%$ se encuentra dentro de la reserva.

Está constituida por las áreas de drenaje pobre localizadas entre las inmediaciones de los ríos Marañón y U cayali, generalmente sujetas a inundación durante las épocas de creciente.

Los suelos que integran estas categorías son los suelos Gramalote, en sus fases de unidad fisiográfica de islas y complejo de orillares reciente y suelo San Ramón en su fase de unidad fisiográfica de orillares subrecientes y antiguos.

Actualmente esta zona se encuentra sometida a extracción selectiva de madera de valor comercial, lo que ha determinado una disminución en calidad como bosque de producción maderera.

\section{Subclase F 2se}

Comprende una superficie de 218,209 ha, que representa el $6.7 \%$ del área evaluada; de los cuales el $46 \%$ pertenece a la reserva. Agrupa tierras de calidad agrológica media, que requieren de prácticas moderadas de manejo y conservación de suelos.

Sus suelos son profundos y tienen drenaje natural bueno a excesivamente drenados de reacción muy ácida, alta saturación de aluminio y baja fertilidad natural.

El suelo que integra esta categoría es el suelo lomada, en su fase fisiográfica, de colinas y lomadas ligera a moderadamente disectadas. 
A grupa a suelos de topografía accidentada, con limitaciones de tipo edáfico y de relieve.

Teniendo en cuenta que estas tierras se encuentran cubiertas de bosques naturales, se recomienda un manejo racional y sostenido de las especies de interés económico del bosque, evitando en lo posible dejar áreas descubiertas que acentúen la erosión, y en aquellos lugares sobreutilizados es de vital importancia realizar programas de reforestación.

\section{CLASE F 3}

Cubre una superficie aproximada de $1{ }^{\prime} 591,643$ has, que representa el 49.2\% del área total evaluada.

A grupa a tierras de calidad agrológica baja, con mayores limitaciones para una actividad de producción forestal, siendo su principal condición extrema la humedad permanente. En base a estas limitaciones se han identificado las subclases $\mathrm{F} 3$ w y $\mathrm{F} 3 \mathrm{sw}$.

\section{Subclase F 3w}

Ocupa una superficie de 863,574 has, que equivale al $26.7 \%$, del total del área evaluada. El $81 \%$ de los cuales ubicado dentro la reserva. Representa una clase conformada de una vegetación mixta, (conjunto de palmeras asociadas con especies arbóreas forestales hidrofíticas).

Son suelos superficiales con la napa freática próxima a la superficie, de textura media a fina, drenaje natural imperfecto a pobre y de reacción muy fuertemente ácida a fuertemente ácida. Las limitaciones principales están asociadas a los factores de humedad.

El suelo que integra esta unidad es Buenos Aires, en su fase correspondiente a las unidades fisiográficas terrazas bajas y medias de drenaje imperfecto a pobre.

Dadas las características de esta subclase, de tierras cubiertas de vegetación mixta, se recomienda la explotación racional del bosque con repoblamiento de las especies aprovechadas, evitando su tumba, para mantener el equilibrio ecológico. 


\section{Subclase F 3sw}

Ocupa una superficie de 728,069 has, que representa el $22.5 \%$ del total del área evaluada. Encontrándose alrededor del $68 \%$ dentro de la reserva. Esta subclase generalmente está constituida por vegetación de palmeras hidrofíticas.

Incluye a suelos, muy superficiales, con la napa freática muy próxima o en la superficie del suelo, la textura es fina; el drenaje natural muy pobre y de reacción de extrema a muy fuertemente ácida, su limitación principal es el exceso de humedad.

El suelo que integra esta unidad es el denominado Tipishca, en su fase fisiográfica de terraza baja y media de drenaje natural muy pobre.

La limitación más importante se encuentra en la humedad constante, debido a su muy pobre drenaje, y al factor edáfico por el bajo nivel de fósforo y alto contenido de aluminio.

Estos suelos no aptos para la agricultura pueden ser revalorados en base al manejo racional del bosque; para lo cual es necesario difundir técnicas de cosecha de frutos, sin derribar el árbol, raleo de los individuos machos y protección de la regeneración natural.

\section{d) Tierras de protección $(X)$}

Estas tierras comprenden una superficie aproximada de 870,192 has; que representa el $26.9 \%$, del área total evaluada, incluyendo 146,718 has. de ríos, meandros, lagunas y localidades. A grupa a aquellas tierras que presentan severas limitaciones para el desarrollo de las actividades agropecuarias y forestales, siendo apropiadas como áreas de protección.

Dentro de este grupo de capacidad de uso mayor, no se considera a clases ni subclases, pero si es necesario indicar el tipo de limitación que restringe su uso. Se ha reconocido las siguientes unidades: 


\section{Símbolo Xw}

Cubre una superficie de 144,468 has, que representa el $4.5 \%$ del área evaluada, con el $77 \%$ del área dentro de la reserva. Son suelos anegados con drenaje pobre a muy pobre y de textura fina.

El manejo de estas tierras debe estar orientado al mantenimiento de la cobertura arbórea y arbustiva existente, con el objeto de conservar el suelo y el hábitat de la fauna terrestre y acuática, evitando su erosión.

\section{Símbolo Xiw}

Cubre una superficie de 579,006 has, que representa el $17.9 \%$ del total del área, con el 62\% dentro de la reserva. A grupa áreas depresionadas, cuyos suelos son de textura fina (Impermeable), con alto nivel freático, producto de las aguas subterráneas.

El manejo y uso de estas tierras debe estar orientado al mantenimiento de la cobertura vegetal natural, hábitat de la fauna silvestre; proporcionando protección a las condiciones ambientales de la zona.

\section{Símbolo X*}

Esta unidad abarca una superficie de 146,718 has, que representa el $4.5 \%$ de la superficie total estudiada, con el $86 \%$ fuera de la reserva. Está formada principalmente por las áreas ocupadas por ríos, lagunas, meandros abandonados y localidades. Deben ser manejados únicamente con fines de protección de los recursos hidrobiológicos y de los valores turísticos en beneficio de los intereses comunes de la colectividad.

\subsection{Características de los Suelos y Capacidad de Uso M ayor de la Tierra Según Unidades Fisiográficas}

El estudio fisiográfico se ha realizado a un nivel de reconocimiento, teniendo como objetivo principal la identificación y delimitación de las formas de tierra dominantes, existentes en la Reserva Nacional Pacaya-Samiria y en el área de su influencia. Esta información ha servido de base para el estudio de evaluación de suelos y de capacidad de uso mayor de las tierras. 
Según el método de análisis fisiográfico utilizado se han identificado dos (2) gran paisajes, tres (3) paisajes, tres (3) subpaisajes y quince (15) unidades fisiográficas, las mismas que se aprecian en el Cuadro $\mathrm{N}$ ㅇ 4.

El primer gran paisaje, "llanura aluvial", agrupa geoformas originadas por la dinámica fluvial de los ríos Marañón y U cayali principalmente y está conformada por sedimentos recientes y subrecientes. Comprende las siguientes unidades fisiográficas: islas, meandros, complejo de orillares, terrazas bajas y medias con diferentes tipos de drenaje, así como pantanos.

El segundo gran paisaje "colinoso", constituido por sedimentos del terciario, posee una topografía accidentada y agrupa una unidad fisiográfica denominada: colinas y lomadas ligera o moderadamente disectadas.

\section{a) Gran Paisaje L lanura Aluvial}

Se caracteriza por presentar una topografía plana a ligeramente ondulada con una pendiente de $0-2 \%$. Este escenario está conformado por las Ilanuras fluviales de los ríos M arañón y U cayali y sus tributarios.

\section{Paisaje L lanura Fluvial Reciente}

Se caracteriza por presentar formas de tierra cuyo origen reciente está sujeta a la dinámica fluvial de los ríos U cayali y Marañón. Soportan inundaciones ocurridas por las crecientes normales de los ríos.

Litológicamente están formados por materiales del cuaternario reciente (Holoceno) constituidos por sedimentos fluviónicos que han sido depositados en forma periódica durante las crecientes de los ríos.

Comprende el subpaisaje llanura de inundación, con los siguientes el ementos:

- Islas, (Símbolo en el mapa Is).

Las islas, son masas de tierra rodeadas de agua, ubicadas en los cauces actuales y abandonados de los ríos U cayali y Marañón. A grupan formas de tierras que son inundables periódicamente en crecientes normales de los ríos, como las playas y barriales. Estas formas de tierras han sido formadas por la propia dinámica de los ríos, las mismas que están sujetas a procesos de erosión y sedimentación. 
L as islas sólo cubren una superficie de 56,926 has (1.76\%), de los cuales el $84 \%$ se localizan en la parte externa de la RNPS. Sus suelos tienen las mismas características que aquellos de los complejos de orillares.

Las islas, que están cubiertas de vegetación arbórea y arbustiva, presentan una relativa estabilidad en relación a aquellas de formación reciente que son formas de tierra bastante inestables y a veces transitorias, que se desplazan anualmente debido a los cambios del cauce de los ríos.

Debido a la relativa estabilidad de algunas islas, parte de ellas es utilizada para la agricultura, principalmente con cultivos de especies de ciclo vegetativo corto, tales como maíz, arroz, caupí, maní, verduras y algunas veces yuca y plátano.

Por el tiempo de formación, se distinguen dos tipos de islas, las de origen reciente y las de origen antiguo.

En las islas de origen reciente predominan dos tipos de suelos. En las partes altas (restingas) se encuentran los tropofluvents, que se caracterizan por no presentar horizontes de diagnósticos, generalmente de textura media y sujetos a la influencia del hidromorfismo en algunas capas inferiores a los $100 \mathrm{~cm}$ de profundidad, sin estructura, de reacción neutra, con bajo contenido de materia orgánica, niveles medios a bajos de fósforo disponible, alta saturación de bases y de buena fertilidad natural.

En cambio, los tropic fluvaquents, que se ubican en las partes más depresionadas (bajiales), se caracterizan por presentar drenaje natural imperfecto a pobres, de textura media a fina, de reacción neutra, alta saturación de bases, bajo contenido de materia orgánica y fósforo, lo que determina una fertilidad natural media.

De acuerdo a su capacidad de uso mayor de las tierras, en las islas de origen reciente se encuentra la Asociación Xiw- $\mathrm{F} 2 \mathrm{w}-\mathrm{A} 3 \mathrm{i}$, que están compuestas generalmente por tres grupos de tierras. EI 70\% de la asociación cubre tierras de protección con drenaje muy deficiente y sujetas a problemas de inundación periódica, $15 \%$ del área representa tierras aptas para producción forestal de calidad agrológica media relacionadas con erosión lateral, y el 15\% de tierras aptas para cultivo en limpio, de calidad agrológica baja, cuyo principal factor limitante es la inundación. 


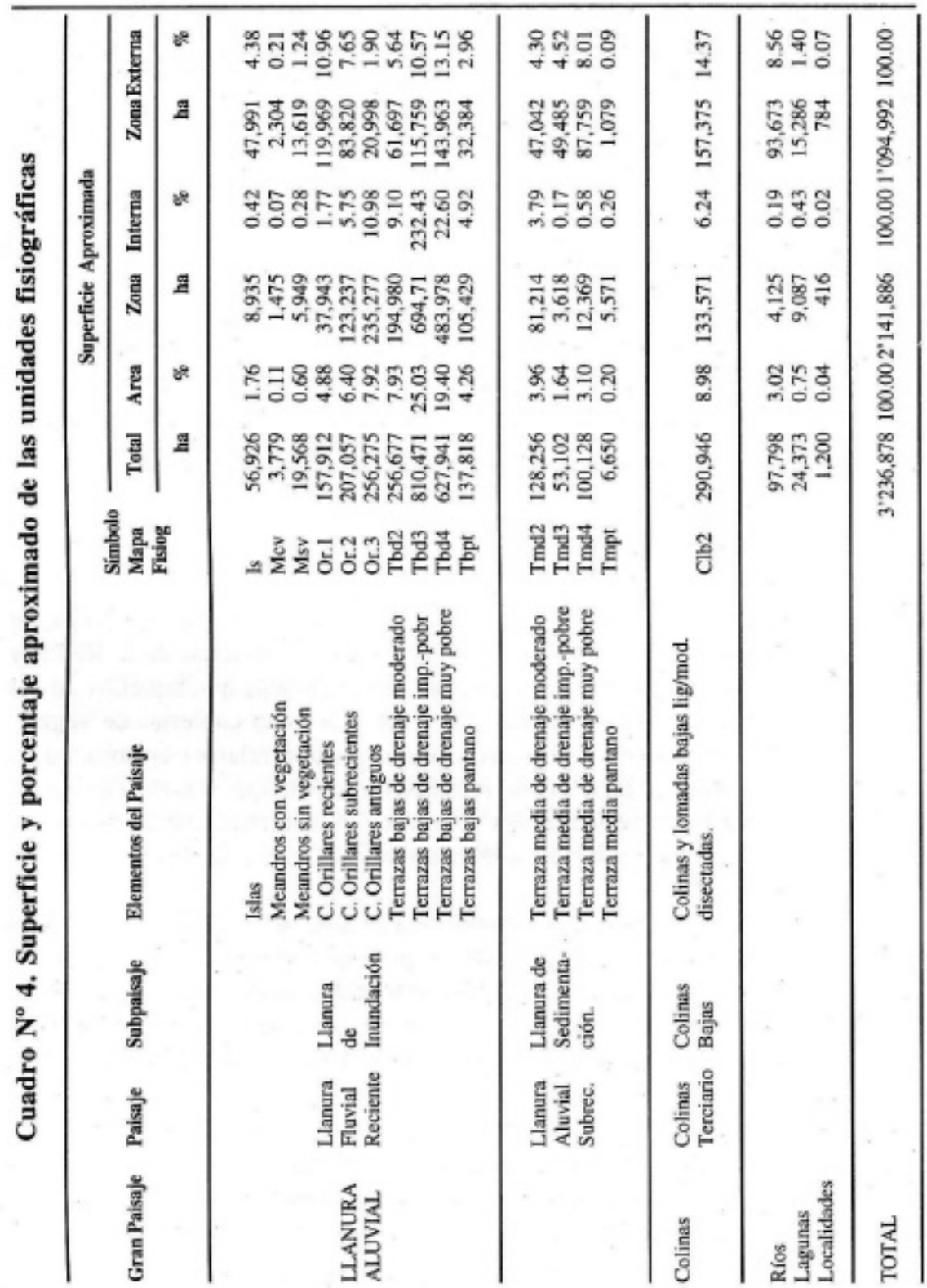


En las islas de origen antiguo también se encuentran dos tipos de suelos. En las partes más altas (restingas), predominan los typic eutropepts, que se caracterizan por presentar perfiles del tipo $A B C$, con textura que varía de media a fina, con reacción ligeramente ácida, con niveles bajo a medio de materia orgánica y fósforo disponible, alta saturación de bases y buena fertilidad natural.

En cambio, en las partes con mayor depresión (bajiales) se encuentran los typic tropaquets, que se caracterizan por presentar una reacción extremadamente ácida, con bajos niveles de materia orgánica y fósforo disponible, al to contenido de aluminio y por consiguiente de fertilidad natural baja.

En estas islas de origen antiguo, se encuentra la A sociación Xiw-F 2 w-A 3i. El $60 \%$ del área corresponde a tierras de protección, 20\% para producción forestal y $20 \%$ para cultivo en limpio.

- M eandros con vegetación (Símbolo M cv)

Esta unidad, que posee sólo una superficie de 3,779 has $(0.11 \%)$, está constituída por pequeños sectores del antiguo cauce del río, que han quedado abandonados por cambios en la posición del río, localizados cerca a él, tienen forma de media luna y se encuentran parcialmente cubiertos de agua y se caracterizan por presentar vegetación, generalmente flotante.

- Meandros sin vegetación (Símbolo M sv)

Es una unidad con características idénticas a la anterior unidad, pero sin vegetación encontrándose en una fase más reciente de desarrollo. Cubre una superficie de 19,568 has $(0,6 \%)$. 
- Complejo de orillares

Constituyen geoformas localizadas principalmente a orillas del río U cayali y M arañón, y representa una superficie significativa del área estudiada (19.2\%), con 621,244 has.

Se originan cuando se rompe el equilibrio erosión-sedimentación, y predomina la erosión lateral en el período de grandes crecientes: el agua se expande por la llanura de inundación, muchas veces en forma turbulenta ocasionando mayor sedimentación por pérdida de velocidad de flujo y formándose tierras más altas denominadas restingas que tienen forma de barras semilunares.

Estas alteran con las partes bajas suavemente curvadas (bajiales), y con las tahuampas que son tierras con drenaje muy pobre. Bajo un criterio adecuado de manejo es posible aprovechar el $40 \%$ de estas formaciones, con cultivos de ciclo corto.

- Complejo de orillares recientes (Símbolo Or1).

Son formas de tierra que se ubican en las orillas convexas como consecuencia de la erosión natural de los ríos, las mismas que sufren inundaciones periódicas.

Poseen una superficie de 157,912 has (4.88\%), de los cuales el $76 \%$ se localizan en la zona externa de la RN PS.

El complejo de orillares recientes esta constituido por formas de tierra reciente, menos desarrolladas por estar sujetas a las inundaciones periódicas y de dimensiones pequeñas, cuya vegetación predominante es herbácea y arbustiva, donde aparecen generalmente las playas y barriales, que pueden tener el mismo uso que las islas.

En esta unidad fisiográfica predomina dos tipos de suelos. En las partes altas (restingas) se encuentran los tropo fluvents, que se caracterizan por no presentar horizontes de diagnósticos, generalmente de textura media y sujetas a la influencia del hidromorfismo en algunas capas inferiores a los $100 \mathrm{~cm}$. de 
profundidad, sin estructura, de reacción neutra, con bajo contenido de materia orgánica, niveles medios a bajos de fósforo disponible, al ta saturación de bases y de buena fertilidad natural.

En cambio, los tropic fluvaquents, que se ubican en las partes más depresionadas (bajiales), se caracterizan por presentar drenaje natural imperfecto a pobres, de textura media a fina, de reacción neutra, alta saturación de bases y bajo contenido de materia orgánica y fósforo, lo cual determina una fertilidad natural media.

Según la capacidad de uso mayor de la tierra, en esta unidad se encuentra la Asociación Xiw-F2w-A3i, que están compuestas generalmente por tres grupos de capacidad de uso mayor.

El $70 \%$ de la asociación cubre tierras de protección con drenaje muy deficiente y sujetas a problemas de inundación periódica, 15\% del área representan tierras aptas para producción forestal de calidad agrológica media relacionadas con erosión lateral, y el 15\% de tierras aptas para cultivo en limpio, de calidad agrológica baja, cuyo principal factor limitante es la inundación.

- Complejo de orillares subrecientes (Símbolo Or2)

Esta formación se ubica en el nivel intermedio entre los orillares recientes y antiguos, caracterizado por presentar mayor desarrollo pedogenético que los primeros, así como de vegetación (arbórea), donde excepcionalmente las inundaciones alcanzan a cubrir totalmente. Poseen una superficie de 207,057 has $(6.4 \%)$, de los cuales el $60 \%$ se ubican en la zona interna de la R N PS.

Esta formación, dentro de los complejos de orillares, es el área más aprovechada en el desarrollo de la agricultura, principalmente con el cultivo de frutales como papaya, mango, guaba, guayaba, poma rosa, taperibá, pan del árbol, plátanos y otros.

En esta unidad fisiográfica se encuentran dos tipos de suelos. En las partes más altas (restingas) están los typic eutropepts, que se caracterizan por presentar perfiles del tipo $A B C$, con textura que varía de media a fina, con reacción ligeramente ácida, con niveles 
bajo a medio de materia orgánica y fósforo disponible, alta saturación de bases y buena fertilidad natural.

En cambio, en las partes con mayor depresión (bajiales) se encuentran los typic tropaquets, que se caracterizan por presentar una reacción extremadamente ácida, con bajos niveles de materia orgánica y fósforo disponible, alto contenido de aluminio y por consiguiente de fertilidad natural baja.

De acuerdo a la capacidad de uso mayor de las tierras, en esta unidad fisiográfica se encuentra la Asociación Xiw-F 2w-A3i. El 60\% del área corresponde a tierras de protección, 20\% para producción forestal y $20 \%$ para cultivo en limpio.

- Complejo de orillares antiguos (Símbolo Or3)

Este complejo posee las mismas características de origen que los descritos anteriormente, diferenciándose por ser más antiguo y tener vegetación de bosque clímax.

Estos orillares se localizan tanto cerca como distante al curso actual del río siendo estos últimos formados en posiciones anteriores.

A medida que el río ha ido cambiando de posición estas barras semilunares han quedado aisladas, careciendo por lo general de agua aunque su drenaje todavía es visible.

Cubren una superficie de 256,275 has (7.9\%), de las cuales el $92 \%$ se localiza en la zona interna de la R NPS.

Son aprovechables en la medida de su cercanía a la orilla del río, del mismo modo que los subrecientes.

En esta unidad fisiográfica se encuentran dos tipos de suelos. En las partes más altas se localizan los typic eutropepts, que se caracterizan por presentar perfiles del tipo ABC, con textura que varía de media a fina, con reacción ligeramente ácida, con niveles bajo a medio de materia orgánica y fósforo disponible, alta saturación de bases y buena fertilidad natural. 
En cambio, en las partes con mayor depresión (bajiales) se encuentran los typic tropaquets, que se caracterizan por presentar una reacción extremadamente ácida, con bajos niveles de materia orgánica y fósforo disponible, alto contenido de aluminio y por consiguiente de fertilidad natural baja.

Según la capacidad de uso mayor de las tierras, la Asociación XiwF 2w-A3i predomina en esta unidad fisiográfica. El $60 \%$ del área corresponde a tierras de protección, $20 \%$ para producción forestal y $20 \%$ para cultivo en limpio.

\section{- Terrazas bajas}

Están Constituida por tierras de topografía plana, con pendiente de $0-2 \%$, con microondulaciones. Localizadas a lo largo de los ríos U cayali y M arañón, y en el interior de la reserva.

Debido a su poca diferencia con el nivel del río sufren inundaciones en la época de crecientes normales. Están constituidas por sedimentos fluviales recientes, y es utilizado para la agricultura.

L as terrazas bajas son las unidades fisiográficas de mayor significado espacial, pues representan cerca del 57\% (1'832,907 has) de la zona de estudio, de los cuales el $81 \%$ se localiza dentro de la RNPS.

- Terrazas B ajas de Drenaje M oderado (Símbolo Tbd2).

Cubren una superficie de 256,677 has ( $7.9 \%$ ), de las cuales el $76 \%$ se localiza dentro de la RNPS. Es una unidad fisiográfica similar a la anterior, pero con drenaje moderado, por los que son aptas para desarrollar actividades agrícolas.

En esta unidad fisiográfica predomina el suelo fluventic eutropepts, que se caracteriza por derivar de sedimentos fluviales subrecientes, presentan perfiles del tipo AC, con epipedón ócrico, y en algunas zonas limitados por el nivel freático (fluctuante según el nivel del agua del río), de colores pardo grisáceo muy oscuro a pardo fuerte, textura media a moderadamente fina, moderadamente drenados, 
ocasionalmente presentan perfiles con uno o más horizontes en avanzado estado de gleizamiento, de reacción neutra, alta saturación de bases y fertilidad natural media a alta.

Según la capacidad de uso mayor de la tierra, la Asociación A3i-Xiw predomina en esta unidad fisiográfica. Esta asociación está conformada por dos grupos de tierras con diferente aptitud de capacidad de uso mayor.

El $60 \%$ presenta vocación para cultivo en limpio con adecuado uso de tecnologías de manejo, por corresponder a suelos de baja calidad agrológica con problemas periódicos de inundación (el $76 \%$ del área está dentro de la reserva). El otro $40 \%$ tiene aptitud sólo para protección de suelo, flora y fauna silvestre.

\section{- Terrazas bajas de drenaje imperfecto a pobre (Símbolo Tbd3).}

Son similares a la unidad fisiográfica antes descrita, diferenciándose por su tipo de drenaje, de imperfecto a pobre. Representan el 25 del área estudiada, de los cuales el $86 \%$ se ubica en la juridicción de la RNPS.

En esta unidad fisiográfica predomina el suelo typic dystropepts, que se caracteriza por ser derivados de materiales aluviales antiguos, de textura fina a moderadamente fina, con perfiles del tipo $A B C$, con profundidad efectiva de más de $90 \mathrm{~cm}$, limitando su profundidad capas arcillosas regularmente compactadas de coloración gris, de textura arcillosa a franco arcillo arenosa, reacción muy fuertemente ácida, con alta saturación de aluminio, y baja fertilidad natural.

La capacidad de uso mayor de la tierra de esta unidad fisiográfica está relacionada con la Consociación F3w, que está conformada principalmente por un grupo de tierras con capacidad de uso mayor para producción forestal; considerada de baja calidad agrológica por presentar serias deficiencias con relación al drenaje. 
- Terraza baja de drenaje muy pobre (Símbolo Tbd4).

Son similares a las anteriores, esta unidad por su topografía depresionada o plana y por su subsuelo impermeable, presenta una condición de mal drenaje lo que se manifiesta por la acumulación de aguas de lluvias y las inundaciones de los ríos. Poseen una superficie de 627,941 has (19\%), de los cuales el 77\% está dentro de la RNPS.

En esta unidad fisiográfica predomina el suelo typic hidroquents, que se caracteriza por ser derivados de materiales aluviales antiguos, de textura media, con perfiles del tipo AC, carecer de estructura, presentar procesos de gleysamiento, de color gris verduzco variando a gris claro, de reacción muy fuertemente ácida, con alta saturación de bases y niveles medios de fósforo disponible que determina una fertilidad natural media.

En estas zonas predomina la vegetación mixta y de palmeras especialmente el "aguaje" (M auritia flexuosa).

En esta unidad fisiográfica se encuentra la Consociación F3sw, que está conformada exclusivamente por grupo de tierras con capacidad de uso mayor de producción forestal, de baja calidad agrológicas por presentar humedad constante, debido al drenaje natural muy pobre y al factor edáfico por el nivel bajo de fósforo y alto contenido de aluminio.

- Terraza baja pantano (Símbolo Tbpt)

Son áreas depresionadas, cuyos suelos son de material fino (impermeables), con alto nivel de aguas subterráneas, contienen materia orgánica parcialmente descompuesta y el agua estancada es de color turbia, la vegetación es hidrofítica, compuesta predominantemente por aguaje (M auritia flexuosa).

Cubren una superficie de 137,818 has (4.26\%), de las cuales el $76 \%$ está dentro de la RNPS. Son de aptitud para protección.

En esta unidad fisiográfica predomina el suelo hydric tropofibrists, que se caracteriza por ser originados a partir de restos de especies vegetales hirofíticas descompuestas en un ambiente de hidromorfía 
permanente, presentan perfiles tipo AC con epipedones hísticos que varía de 0.5 a $2.5 \mathrm{~m}$ que descansa sobre un material arcilloso muy gleisado, el drenaje natural es muy pobre, presentan reacción fuertemente ácida y alta capacidad de intercambio catiónico, debido fundamentalmente al elevado contenido de materia orgánica.

Según su capacidad de uso mayor de las tierras, en esta unidad se encuentra la Consociación Xw, que está conformada exclusivamente por tierras con capacidad de uso mayor para protección por estar ubicados en áreas exclusivamente pantanosa.

\section{Paisaje de Ilanura aluvial subreciente}

Está constituido por sedimentos aluviales pleistocénicos, de topografía plana a ligeramente ondulada y comprende terrazas de segundo nivel no inundable en crecientes normales.

Comprende el subpaisaje Ilanura de sedimentación, la que está constituida de terrazas medias que han alcanzado una altura suficiente que no permiten que sean inundados durante las épocas de crecientes normales.

L a topografía generalmente varía de plana a ligeramente ondulada y sólo cubre una superficie de 288,137 has. Se distribuye en la parte occidental de la RNPS, y en mayor proporción en la margen derecha del río Ucayali y en la margen izquierda del río Marañon. Comprende los siguientes elementos:

- Terrazas medias de drenaje moderado (Símbolo Tmd2).

Son terrazas de segundo nivel, que se caracterizan por tener un drenaje moderado, con suelos por lo general de textura gruesa a media. Poseen una superficie de 128, 256 has (3.96\%).

En esta unidad fisiográfica predominan los suelos Oxic Dystropepts y Typic Hapludults, que se caracterizan por ser originados a partir de depósitos aluviales antiguos y de materiales residuales, estos presentan un horizonte argílico profundo, de color que varía de gris 
claro a pardo y rojo amarillento, de textura media a fina, de reacción muy fuertemente ácida, al ta saturación de aluminio, bajo contenido de materia orgánica y fósforo a través del perfil, y por consiguiente una fertilidad natural baja.

La capacidad de uso mayor de las tierras de esta unidad está relacionadas con la Asociación C 3s-Xiw, que comprende tierras aptas para cultivo permanente en un $60 \%$, y áreas destinadas a protección en un $40 \%$. Esta asociación incluye suelos de baja capacidad agrológica con problemas moderados de drenaje. El 63\% está dentro de la reserva.

- Terrazas medias de drenaje imperfecto a pobre (Símbolo Tmd3).

Presenta características similares a las anteriormente descritas, diferenciándose por el tipo de drenaje. Cubren sólo 53,102 has $(1.6 \%)$.

En esta unidad fisiográfica predomina el suelo Typic Dystropepts, que derivar de materiales aluviales antiguos, de textura fina a moderadamente fina, con perfiles del tipo $A B C$, con profundidad efectiva de más de $90 \mathrm{~cm}$, limitando su profundidad capas arcillosas regularmente compactadas de coloración gris, de textura arcillosa a franco arcillo arenosa, reacción muy fuertemente ácida, con alta saturación de aluminio y baja fertilidad natural.

La capacidad de uso mayor de la tierra está relacionada con la Consociación $\mathrm{F} 3 \mathrm{w}$, que está conformada por un grupo de tierras con capacidad de uso mayor para producción forestal en el 100\%; consideradas de baja calidad agrológica por presentar serias deficiencias con relación al drenaje.

- Terrazas medias de drenaje muy pobre (Símbolo Tmd4).

Este tipo de terrazas presentan superficies plano-concavas; con similares características que la unidad fisiográfica de terraza baja de drenaje muy pobre. 
Generalmente ocupan las áreas depresionadas de las terrazas medias. Poseen una superficie de 100,129 has (3.1\%).

En esta unidad fisiográfica se encuentra el suelo Typic Tropaquepts, que se caracteriza por presentar una reacción extremadamente ácida a muy fuertemente ácida, con bajos niveles de materia orgánica y fósforo disponible y al to contenido de aluminio, con lodo por debajo de los $40 \mathrm{~cm}$, lo que no permite ningún tipo de explotación agropecuaria.

La capacidad de uso mayor de las tierras está relacionada con la Consociación $\mathrm{F} 3 \mathrm{sw}$, que está conformada exclusivamente por grupo de tierras con capacidad de uso mayor de producción forestal, de baja calidad agrológicas por presentar humedad constante debido al drenaje natural muy pobre que exhiben y al factor edáfico.

\section{- Terrazas medias pantanos (Símbolo Tmpt)}

Es una unidad fisiográfica similar a la que se informó en terrazas bajas, diferenciándose en que ésta se encuentra en las terrazas medias. Cubre una superficie pequeña de 6,650 has $(0.2 \%)$.

El suelo predominante corresponde a Hydric Tropofibrists, que se caracteriza por ser originados a partir de restos de especies vegetales hidrofíticas descompuestas en un ambiente de hidromorfía permanente, la vegetación está compuesta predominantemente por aguaje (Mauritia flexuosa), asociado con otras especies hidrofíticas, presentan perfiles tipo AC con epipedones hísticos que varía de 0.5 a $2.5 \mathrm{~m}$ y que descansa sobre un material arcilloso muy gleisado.

En esta unidad fisiográfica se encuentra la Consociación Xw, que está conformada exclusivamente por tierras con capacidad de uso mayor para protección por estar ubicados en áreas exclusivamente pantanosas. 


\section{b) Gran paisaje colinoso}

Este escenario fisiográfico comprende el siguiente paisaje:

\section{Paisaje colinoso del terciario}

Está representado por colinas bajas y lomadas, de relieve ondulado a fuertemente ondulado con alturas que oscilan entre 30-45 m, con respecto al nivel de base local. La génesis de esta superficie se debe a movimientos orogénicos y tectónicos complementados con la acción modeladora de la erosión hídrica sobre la antigua Ilanura aluvial.

Litológicamente está conformada por materiales sedimentarios del terciario (arcillitas, lutitas, areniscas, lodolitas) lo que permitió la conservación de las colinas, este paisaje comprende el subpaisaje colinas y lomadas, que está formado por la siguiente unidad fisiográfica:

- Colinas y lomadas bajas ligera a moderadamente disectadas (Símbolo Clb2)

Las colinas bajas presentan un relieve de ligera a moderadamente disectadas, teniendo pendientes que oscilan entre 15 y $45 \%$.

Las lomadas poseen superficies más onduladas y de contornos suaves y redondeados, presentan altura hasta de 30 metros en relación a la base y en laderas de pendientes que oscilan entre 15-25\%. Poseen una superficie de 290,946 has $(8.98 \%)$.

El suelo predominante en esta unidad corresponde a Typic Hapludults, que se caracteriza por presentar un perfil $A B C$, con epipedón ócrico y horizonte argílico profundo, de color que varía de pardo a rojo amarillento, de textura media a fina, de drenaje natural algo excesivo en las pendientes pronunciadas, de reacción muy fuertemente ácida, alta saturación de aluminio, bajo contenido de materia orgánica y fósforo a través del perfil, y por consiguiente posee una fertilidad natural baja. 
En esta unidad fisiográfica se encuentra la Asociación F 2se-C 3se, que comprende dos grupos de tierras: tierras aptas para producción forestal, consideradas de calidad agrológica media por presentar deficiencias de carácter edáfico en un $75 \%$ y de tierras aptas para cultivo permanente de capacidad agrológica baja con técnicas apropiadas de manejo en $25 \%$.

Estas tierras se ubican en las colinas bajas y lomadas ligeramente disectadas con pendiente menores de $25 \%$ susceptibles a problemas de erosión de ambas márgenes de los ríos U cayali y M arañón y en el límite occidental de la reserva.

\section{c) Áreas misceláneas}

Comprenden áreas que ocupan ríos, lagunas, cochas y diferentes localidades como caseríos y pueblos, entre los cuales tenemos a Requena, $\mathrm{N}$ auta y Jenaro Herrera, entre los ríos $M$ arañón, U cayali y Tigre.

E stas áreas abarcan una superficie de 123,371 has (3.81\%). 


\section{BIBLIOGRAFIA}

BAYLEY, P.; R. VASQUEZ,; P. GHERSI; P. SOINI y M. PINEDO. 1992. Enviromental review of the Pacaya Samiria National Reserve in Perú and Assesment of Project (527-0342). N ature Conservancy - USA. 81 p.

CENTRO DE DATOS PARA LA CONSERVACION. UNIVERSIDAD NACIONAL AGRARIA LA MOLINA. 1994. Evaluación Ecológica de la Reserva Nacional Pacaya-Samiria. Informe presentado a la Fundación Peruana para la Conservación de la Naturaleza y The Nature Conservancy. Employment and $\mathrm{N}$ atural Resources Sustainability Project.

COREPASA. 1986. Plan Maestro de la Reserva Nacional Pacaya Samiria. Edición Imprenta DESA. 239 p.

HOAG, R. 1985. Characterization of soil on floodplains of tributaries flowing into the amazon river in Perú. 118 p. (Tesis Doctoral en preparación)

IIAP. 1987. Estudio de suelos aluviales: zona Tamshiyacu-Indiana. Ed. IIAP- Iquitos. $50 \mathrm{p}$.

IIAP. 1994. Estudio socioeconómico de las poblaciones vecinas de la Reserva Nacional Pacaya-Samiria. Convenio IIAP-FPCN. Informe Técnico.

MINISTERIO DE AGRICULTURA. 1975. Reglamento de Clasificación de Tierras. Lima 17 p. (mimeografiado).

ONERN. 1975. Inventario, evaluación e integración de los Recursos Naturales de la zona Iquitos, Nauta, Requena y Colonia Angamos. Lima 334 p.

ONERN. 1976. Inventario, evaluación e integración de los Recursos Naturales de la selva. Lima $289 \mathrm{p}$.

ONERN .1982. Clasificación de tierras del Perú. Lima, 185 p.

ONERN. 1984. Inventario y evaluación de los Recursos Naturales de la Microrregión Pastaza - Tigre. Lima, 288 p. 
ONERN - IIAP. 1984. Mapa fisiográfico del sector de suelos inundables entre la boca del río Pachitea y la ciudad de Nauta. Lima. $14 \mathrm{p}$.

RODRIGUEZ, F., P. VASQUEZ Y M. RODRIGUEZ, 1995. La Reserva Nacional Pacaya-Samiria: Análisis Integrado. FPCN. 84 p.

SOIL SURVEY STAFF. 1990. Keys to Soil Taxonomy, Fourt edition. SMSS technical monograph $\mathrm{N}$ ㅇ. 6. Blacksburg, Virginia. $423 \mathrm{p}$.

UNA. 1984. Inventario, Evaluación e Integración de los Recursos Naturales de la zona de Contamana, Pampa Hermosa y O rellana. U niversidad $\mathrm{N}$ acional A graria L a M olina. Lima - Informe de avance. 146 p. 


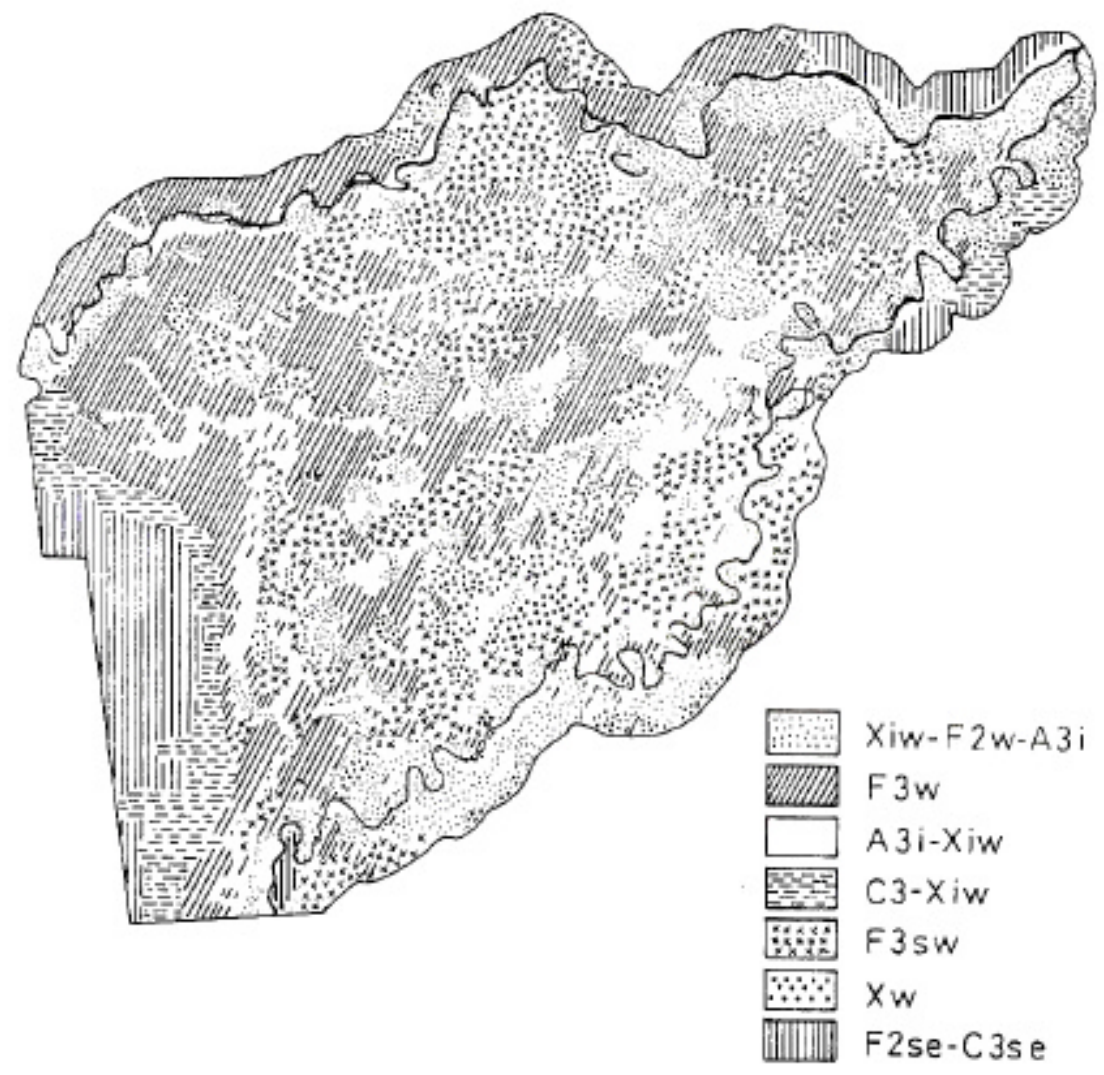

PACAYA-SAMIRIA

CAPACIDAD DE USO DE SUELO 